\title{
In Search of Proper Pareto-optimal Solutions Using Multi-objective Evolutionary Algorithms
}

\author{
Pradyumn Kumar Shukla \\ Institute of Numerical Mathematics \\ Department of Mathematics \\ Technische Universität Dresden \\ Dresden PIN 01069, Germany \\ pradyumn.shukla@mailbox.tu-dresden.de
}

\begin{abstract}
There are multiple solution concepts in multi-objective optimization among which a decision maker has to select some good solutions usually which satisfy some trade-off criteria's. The need for potentially good solutions has always been one of the primary aims in multiobjective optimization. A complete representation of all these solutions is only possible with population based approaches like multi-objective evolutionary algorithms since then trade-off's can be calculated at each generation from the population members. Thus this paper proposes the use of multi-objective evolutionary algorithms for obtaining a complete representation of these good solutions. Theoretical results show how one can integrate search procedure for obtaining these solutions in population based evolutionary algorithms and some convergence results. Finally simulation results are presented on a number of test problems.
\end{abstract}

Keywords: Multi-objective optimization, Trade-off, Evolutionary algorithms.

\section{Introduction}

Multi-objective optimization is one of the most rapidly growing areas of modern optimization theory, see for example Deb [2], Miettinen [7] and the references therein. Since there are multiple solution concepts in multi-objective optimization it often becomes a challenging issue both in theory and practice. The set of all efficient points as is well known lies in the boundary of the objective space and is thus referred to as the efficient frontier. However all points on the frontier need not have equally nice properties which a decision maker may desire and thus one needs to filter out the bad Pareto points and keep the good ones. Such nice Pareto points are referred to in the literature proper Pareto solutions. Thus the need for potentially good solutions has always been one of the primary aims in multi-objective optimization. Good solutions can be thought of as "knee-points" on the efficient frontier or that are good in trade-off with respect to other solutions. However in most of practical and large scale problems, the user may usually not get the exact efficient front and thus he has to 
be content with approximate solutions. This usually happens if one uses population based approach like Multi-objective Evolutionary Algorithm (MOEA) or any other algorithm. In these algorithm the obtained solutions may be thought of as approximate representation of the efficient front. We need to filter out the bad ones and keep the so called $\varepsilon$-proper Pareto solutions. A complete representation of $\varepsilon$-proper Pareto solutions is only possible using population based algorithms since trade-off's need to be calculated for them. This paper presents a way to obtain all these $\varepsilon$-proper Pareto solutions using MOEA's. Theoretical results are also presented so show convergence of such an algorithm.

The paper has been organized in three sections of which this is the first. Definitions of $\varepsilon$-proper efficiency theoretical results are presented in Section 2 while simulation results and conclusions are presented in Section 3.

\section{Theoretical Results}

Consider the following general multi-objective optimization problem $(M P)$ :

$$
\min _{x \in X} f(x)=\left(f_{1}(x), f_{2}(x), \ldots, f_{m}(x)\right)
$$

where each $f_{i}: \mathbb{R}^{n} \rightarrow \mathbb{R}$ and $X \subseteq \mathbb{R}^{n}$. In what follows we will consider $\varepsilon \in \mathbb{R}_{+}^{m}$, i.e. $\varepsilon=\left(\varepsilon_{1}, \ldots, \varepsilon_{m}\right), \varepsilon_{i} \geq 0$ for all $i$. In some cases we will set $\varepsilon_{i}=\varepsilon^{\prime}$, for all $i$ and then $\varepsilon=e \varepsilon^{\prime}$ where $e=(1, \ldots, 1) \in \mathbb{R}_{+}^{m}$.

Definition $1 \varepsilon$-Pareto optimal. Let $\varepsilon \in \mathbb{R}_{+}^{m}$ be given then a point $x^{*} \in X$ is said to be an $\varepsilon$-Pareto optimal of $(M P)$ if there exists no $x \in X$ such that,

$$
f_{i}(x) \leq f_{i}\left(x^{*}\right)-\varepsilon_{i}, \quad \forall i \in\{1,2, \ldots, m\} .
$$

and with strict inequality holding for at least one index.

Observe that if $\varepsilon=0$, the above definition reduces to that of a Pareto optimal. Let us denote the set of $\varepsilon$-Pareto points as $X_{\varepsilon-\text { par }}$ and the set of Pareto points as $X_{\text {par }}$. The notion of trade-off is inherent in multi-objective optimization which led to more robust solution concept defined next.

Definition 2 Proper Pareto optimal. $x_{0} \in X$ is called proper Pareto optimal if $x_{0}$ is Pareto optimal and if there exists a number $M>0$ such that for all $i$ and $x \in X$ satisfying $f_{i}(x)<f_{i}\left(x_{0}\right)$, there exists an index $j$ such that $f_{j}\left(x_{0}\right)<f_{j}(x)$ and moreover $\left(f_{i}\left(x_{0}\right)-f_{i}(x)\right) /\left(f_{j}(x)-f_{j}\left(x_{0}\right)\right) \leq M$.

There are many other notions of proper Pareto optimal solutions and the above one based on trade-off's was introduced by Geoffrion [5]. Let us denote the set of all Geoffrion properly Pareto optimal solution as $X_{G}$.

Lemma 1. A point $x_{0} \in X_{G}$ if and only if there exists $M>0$ such that the following system is inconsistent (for all $i=1,2, \ldots, m$ and for all $x \in X$ ).

$$
\begin{aligned}
& -f_{i}\left(x_{0}\right)+f_{i}(x)<0 \\
& -f_{i}\left(x_{0}\right)+f_{i}(x)<M\left(f_{j}\left(x_{0}\right)-f_{j}(x)\right) \quad \forall j \neq i .
\end{aligned}
$$


Proof: If $x_{0} \in X_{G}$ then it is clear from the definition that above system is inconsistent.

Suppose the system is inconsistent for some $M>0$. We claim that $x_{0} \in X_{\text {par }}$. If not then on the contrary then there exists $x \in X$ such that $f_{l}(x)<f_{l}\left(x_{0}\right)$ for some index $l$, and $f_{k}(x) \leq f_{k}\left(x_{0}\right)$, for all $k \neq l$. Thus one easily sees that system 1 has a solution for index $i=l$. Hence $x_{0} \in X_{\text {par }}$. If $x_{0} \notin X_{G}$ then for all $\bar{M}>0$ there is an index $i$, and some $x \in X$ satisfying $-f_{i}\left(x_{0}\right)+f_{i}(x)<0$, $-f_{i}\left(x_{0}\right)+f_{i}(x)<\bar{M}\left(f_{j}\left(x_{0}\right)-f_{j}(x)\right)$ for all $j$ such that $-f_{j}\left(x_{0}\right)+f_{j}(x)>0$ (such a $j$ exists since $\left.x_{0} \in X_{\text {par }}\right)$. For $j$ such that $-f_{j}\left(x_{0}\right)+f_{j}(x) \leq 0,-f_{i}\left(x_{0}\right)+f_{i}(x)<$ $\bar{M}\left(f_{j}\left(x_{0}\right)-f_{j}(x)\right)$ is trivially true. Thus the system is consistent for all $\bar{M}>0$, and hence a contradiction.

Note that in Geoffrion's definition $x \in X$. However as shown in next lemma, when $Y=f(X)$ is $\mathbb{R}_{+}^{m}$ compact (i.e. the sections $\left(y-\mathbb{R}_{+}^{m}\right) \cap Y$ are compact for all $y \in Y$ ) then this can be replaced by $x \in X_{\text {par }}$.

Lemma 2. Suppose that $Y=f(X)$ is $\mathbb{R}_{+}^{m}$ compact, then $x_{0} \in X_{G}$ if $x_{0}$ is Pareto optimal and if there exists a number $M>0$ such that for all $i$ and $x \in X_{\text {par }}$ satisfying $f_{i}(x)<f_{i}\left(x_{0}\right)$, there exists an index $j$ such that $f_{j}\left(x_{0}\right)<f_{j}(x)$ and moreover $\left(f_{i}\left(x_{0}\right)-f_{i}(x)\right) /\left(f_{j}(x)-f_{j}\left(x_{0}\right)\right) \leq M$.

Suppose that $x_{0}$ satisfies the conditions of the lemma. Then using Lemma 1, we obtain that for all $\hat{x} \in X_{\text {par }}$ the following system which we mark as (System 1), has no solutions,

$$
\begin{aligned}
& -f_{i}\left(x_{0}\right)+f_{i}(\hat{x})<0 \\
& -f_{i}\left(x_{0}\right)+f_{i}(\hat{x})<M\left(f_{j}\left(x_{0}\right)-f_{j}(\hat{x})\right) \quad \forall j \neq i .
\end{aligned}
$$

Take any $x \in X, x \notin X_{\text {par }}$. Now since $Y=f(X)$ is $\mathbb{R}_{+}^{m}$ compact so there exists $\hat{x} \in X_{\text {par }}$ such that

$$
\begin{array}{rr}
f_{i}(\hat{x})-f_{i}(x) \leq 0 & \forall i=1,2, \ldots, m \\
f_{k}(\hat{x})-f_{k}(x)<0 & \text { for some } k
\end{array}
$$

Since the System 1 has no solutions, thus we obtain that the following system also has no solutions

$$
\begin{aligned}
& -f_{i}\left(x_{0}\right)+f_{i}(\hat{x})<f_{i}(\hat{x})-f_{i}(x) \\
& -f_{i}\left(x_{0}\right)+f_{i}(\hat{x})<M\left(f_{j}\left(x_{0}\right)-f_{j}(\hat{x})\right)+M\left(f_{j}(\hat{x})-f_{j}(x)\right)+f_{i}(\hat{x})-f_{i}(x) \quad \forall j \neq i .
\end{aligned}
$$

which is equivalent to saying that the following system is inconsistent,

$$
\begin{aligned}
& -f_{i}\left(x_{0}\right)+f_{i}(x)<0 \\
& -f_{i}\left(x_{0}\right)+f_{i}(x)<M\left(f_{j}\left(x_{0}\right)-f_{j}(x)\right) \quad \forall j \neq i .
\end{aligned}
$$

Thus System 1 has no solutions for any $x \in X$. Thus $x \in X_{G}$ 
Definition 3 e-properly Pareto optimal (Liu [6]). A point, $x^{*} \in X$ is called $\varepsilon$-proper Pareto optimal, if $x^{*}$ is $\varepsilon$-Pareto optimal and there exists a number $M>0$ such that for all $i$ and $x \in X$ satisfying $f_{i}(x)<f_{i}\left(x^{*}\right)-\varepsilon_{i}$, there exists an index $j$ such that $f_{j}\left(x^{*}\right)-\varepsilon_{j}<f_{j}(x)$ and moreover $\left(f_{i}\left(x^{*}\right)-f_{i}(x)-\right.$ $\left.\varepsilon_{i}\right) /\left(f_{j}(x)-f_{j}\left(x^{*}\right)+\varepsilon_{j}\right) \leq M$.

Observe that if $\varepsilon=0$, the above definition reduces to that of a Geoffrion proper Pareto optimal. Let us denote the set of all Liu properly Pareto optimal solution as $X_{L}(\varepsilon)$. Let us however observe in the above definition and definition $2.2, M$ is arbitrary. On the other side $M$ provides a bound on the trade off between the components of the objective vector. It is more natural to expect in practice the decision maker will provide a bound on such trade offs. Thus we are motivated to define the following.

Definition 4 Geoffrion $M$ properly Pareto optimal. Given a positive number $M>0, x_{0} \in X$ is called Geoffrion $M$ proper Pareto optimal if $x_{0}$ is Pareto optimal and if for all $i$ and $x \in X$ satisfying $f_{i}(x)<f_{i}\left(x_{0}\right)$, there exists an index $j$ such that $f_{j}\left(x_{0}\right)<f_{j}(x)$ and moreover $\left(f_{i}\left(x_{0}\right)-f_{i}(x)\right) /\left(f_{j}(x)-f_{j}\left(x_{0}\right)\right) \leq M$.

Let us denote the set of all Geoffrion $M$ properly Pareto optimal solution as $X_{M}$. It is to be noted that a similar modified definition is also possible for Liu $\varepsilon$-proper Pareto optimal solutions. Let us denote the set of all $M \varepsilon$-proper Pareto optimal as $X_{M}(\varepsilon)$.

Theorem 1. Let $\varepsilon=\varepsilon^{\prime} e$ where $\varepsilon^{\prime} \in \mathbb{R}, \varepsilon^{\prime}>0$ and $e=(1,1, \ldots, 1)$, then for any fixed $M$,

$$
X_{M}=\cap_{\varepsilon^{\prime}>0} X_{M}(\varepsilon)
$$

Proof: Let $x_{0} \in \cap_{\varepsilon^{\prime}>0} X_{M}(\varepsilon)$. Hence for any $\varepsilon^{\prime}>0$, and for all $i$, the following system

$$
\begin{array}{r}
-f_{i}\left(x_{0}\right)+f_{i}(x)+\varepsilon^{\prime}<0 \\
M f_{j}(x)+f_{i}(x)-M f_{j}\left(x_{0}\right)-f_{j}\left(x_{0}\right)+M \varepsilon^{\prime}+\varepsilon^{\prime}<0
\end{array}
$$

has no solutions in $x \in X$ Let $W=\mathbb{R}^{m} \backslash\left(-i n t \mathbb{R}_{+}^{m}\right)$ and consider the vectors $F^{i}(\varepsilon)$ (for all $i=1, \ldots, m$ ) whose first component is given by $-f_{i}\left(x_{0}\right)+f_{i}(x)+\varepsilon^{\prime}$ and whose $j^{\text {th }}$ component is equal to $M f_{j}(x)+f_{i}(x)-M f_{j}\left(x_{0}\right)-f_{j}\left(x_{0}\right)+M \varepsilon^{\prime}+\varepsilon^{\prime}$, for all $j=2, \ldots, m$ then $F^{i}(\varepsilon) \in W$ for all $x \in X$. Now since $W$ is a closed cone for each $i$

$$
\lim _{\varepsilon \rightarrow 0} F^{i}(\varepsilon) \in W
$$

This shows that the following system

$$
\begin{aligned}
-f_{i}\left(x_{0}\right)+f_{i}(x) & <0 \\
M f_{j}(x)+f_{i}(x)-M f_{j}\left(x_{0}\right)-f_{j}\left(x_{0}\right) & <0
\end{aligned}
$$


is inconsistent for all $x \in X$. Thus by Lemma $1 x_{0}$ is $M$-properly Pareto optimal, or $x_{0} \in X_{M}$. This shows that $\cap_{\varepsilon^{\prime}>0} X_{M}(\varepsilon) \subset X_{M}$ Conversely, let $x_{0} \in X_{M}$, thus for all $i=1, \ldots, m$ following system

$$
\begin{aligned}
-f_{i}\left(x_{0}\right)+f_{i}(x) & <0 \\
M f_{j}(x)+f_{i}(x)-M f_{j}\left(x_{0}\right)-f_{j}\left(x_{0}\right) & <0
\end{aligned}
$$

is inconsistent for all $x \in X$, thus the following system

$$
\begin{gathered}
-f_{i}\left(x_{0}\right)+f_{i}(x)<-\varepsilon^{\prime} \\
M f_{j}(x)+f_{i}(x)-M f_{j}\left(x_{0}\right)-f_{j}\left(x_{0}\right)<-M \varepsilon^{\prime}-\varepsilon^{\prime}
\end{gathered}
$$

is also inconsistent for all $x \in X$.Thus $x_{0}$ is $M \varepsilon$-properly Pareto for all $\varepsilon^{\prime}>0$ Hence $x_{0} \in \cap_{\varepsilon^{\prime}>0} X_{M}(\varepsilon)$.
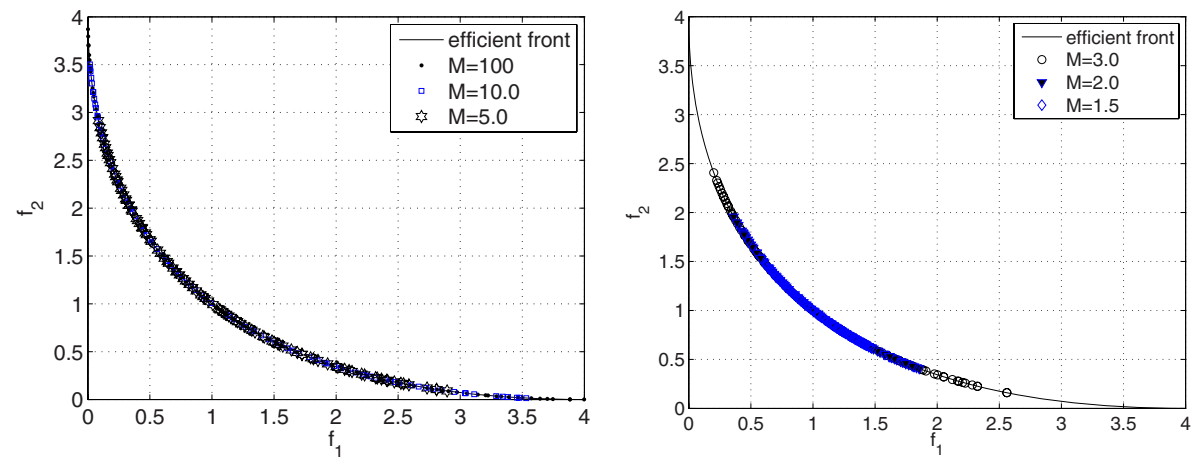

Fig. 1. Proper Pareto optimal solutions Fig. 2. Proper Pareto optimal solutions obtained using $\mathrm{M}=100,10$ and 5 on $\mathrm{SCH}$ obtained using $\mathrm{M}=3,2$ and 1.5 on $\mathrm{SCH}$

Note that for checking an obtained solution for proper Pareto optimality one needs to checks boundedness of trade-off's with all feasible points. Using Lemma 2 however we need to check this for only solutions that belong to the non-dominated front. Thus the above theoretical results can be effectively applied to population based multi-objective evolutionary algorithms since in these algorithms one gets a approximate non-dominated front at each iteration. Theorem 1 says that if an algorithm is computing approximate proper Pareto optimal solutions then in the end we obtain exact proper Pareto optimal solutions. In order to compute approximate proper Pareto optimal solutions we take the elitist non-dominated sorting GA or NSGA-II [3] and introduce constraints as follows. Given any solution $f\left(x_{0}\right)$ at any generation, we calculate the following constraint violation

$$
c\left(x_{0}\right)=\sum_{x} \min \left\{0,\left\{M-\left(f_{i}\left(x_{0}\right)-f_{i}(x)\right) /\left(f_{j}(x)-f_{j}\left(x_{0}\right)\right)\right\}\right\}
$$



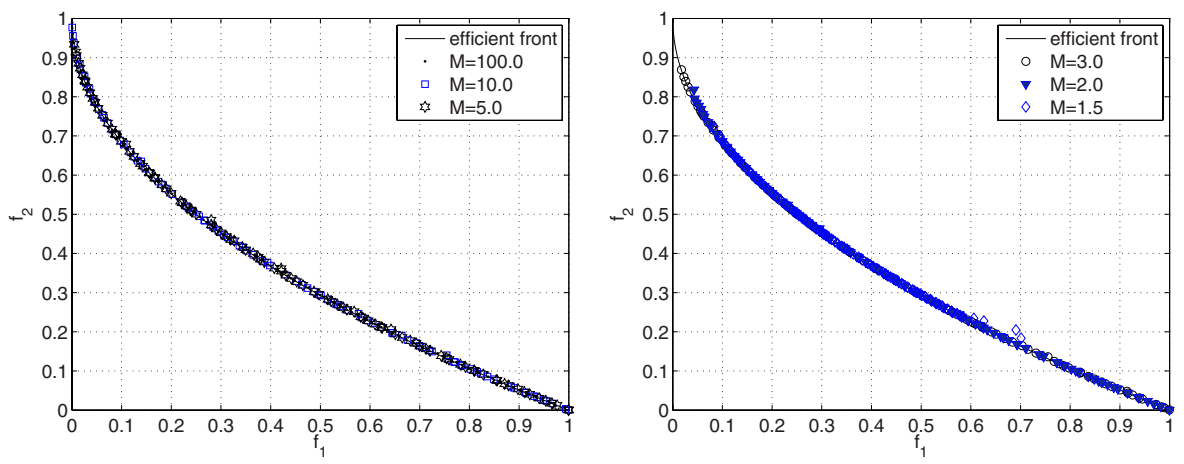

Fig. 3. Proper Pareto optimal solutions Fig. 4. Proper Pareto optimal solutions obtained using $\mathrm{M}=100,10$ and 5 on ZDT1 obtained using $\mathrm{M}=3,3$ and 1.5 on ZDT1

for all solutions $x$ and all indices $(i, j)$ that satisfy $f_{i}(x)<f_{i}\left(x_{0}\right), f_{j}(x)>f_{j}\left(x_{0}\right)$. Thus for any solution that is non Pareto optimal $c\left(x_{0}\right)$ provides a measure of how far is the solution from proper Pareto optimal. For proper Pareto optimal solutions the constraint violation is zero. Thus this provides a measure to penalty solutions that are non Pareto optimal.

\section{Simulation Results and Conclusions}

In this section, we apply the above constraint approach to obtain proper Pareto optimal solutions for different values of $M$. For the NSGA-II, we use a standard real-parameter SBX and polynomial mutation operator with $\eta_{c}=10$ and $\eta_{m}=$ 10, respectively [2] (unless otherwise stated). For all problems solved, we use a population of size 100. We set the number of function evaluations as 20000 for each problems.

First we consider a one variable bi-objective Schaffler's test problem ( $\mathrm{SCH}$ ). This problem is unconstrained and has a convex efficient frontier. The Pareto optimal solutions correspond to $x=[0,2]$. Figure 1 shows the obtained efficient front corresponding to $M=100,10$ and 5. The complete efficient front is also shown. The unrestricted efficient front can be seen as solutions corresponding to proper Proper optimal solutions with $M=\infty$. It can be seen that all the efficient front is proper Pareto optimal with respect to $M=100$. The efficient front reduces when smaller values of $\mathrm{M}$ are used with $f_{1}=0.36$ to 1.89 for $M=2$ (Figure 2).

The box-constrained ZDT1 problem has a convex Pareto optimal front for which solutions correspond to $0 \leq x_{1}^{*} \leq 1$ and $x_{i}^{*}=0$ for $i=2,3, \ldots, 30$. Here Figure 3 and 4 show the obtained efficient front corresponding to different values of $M$. It can be seen that (as opposed to $\mathrm{SCH}$ ) here only the part corresponding to minimization of $f_{1}$ is chopped out as $M$ value is reduced. This is because $M$ proper Pareto optimal solutions are based on trade-off's and are thus 


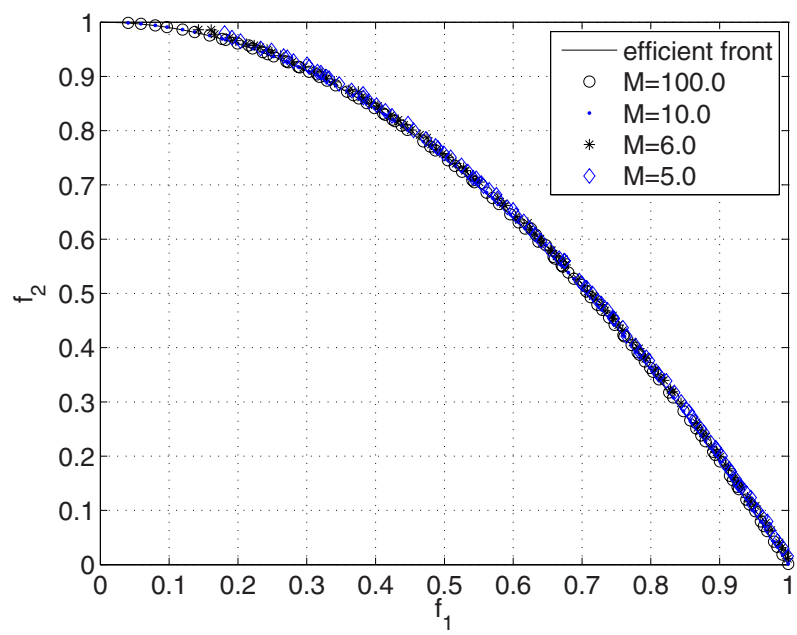

Fig. 5. Proper Pareto optimal solutions obtained using $M=100,10,6$ and 5 on ZDT2

related to slope of efficient front. In the ZDT1 problem slope is more near the $f_{1}$ minimum.

The box-constrained ZDT2 problem has a non-convex Pareto optimal front for which solutions correspond to $0 \leq x_{1}^{*} \leq 1$ and $x_{i}^{*}=0$ for $i=2,3, \ldots, 30$. This problem is non-convex so in this case guided domination approach 1] cannot be used for finding intermediate regions in this problem. However even in this case concept of trade-off can be applied to obtain M proper Pareto optimal solutions. Figure 5 shows the obtained efficient front corresponding to different values of $M$. As opposed to ZDT1 it is observed that for $M$ values smaller than 5.0 only a very small part of efficient front is obtained while no feasible solution is $M$ proper Pareto optimal values for $M=2$. Thus in this case these values give range of realistic trade-off values to a decision maker.

Finally we consider a constrained test problem (CTP7). This problem has disconnected set of continuous regions. As suggested in [4] we use five decision variables. Here the efficient front consists of six disconnected convex regions. Figure 6 and 7 show the obtained efficient front corresponding to different values of $M$. It can be seen that all six parts of efficient front are obtained with $M$ proper Pareto optimal solutions corresponding to $M=100,5$ and 2 . With $M=1.5$ however only four parts remain and for $M=1.2$ only one part of efficient front remains.

The decision maker could also use different $M$ values for trade-off's in different objectives. It is a simple exercise to show the theoretical results presented in Section 2 remain valid in such a case. Finally it is to be noted that the convergence result of Theorem 1 could be applied to any other population based multi-objective evolutionary algorithm other than NSGA-II. In such cases non $M$ proper Pareto optimal solutions could be penalized and then some constraint handling approach can also be used. 

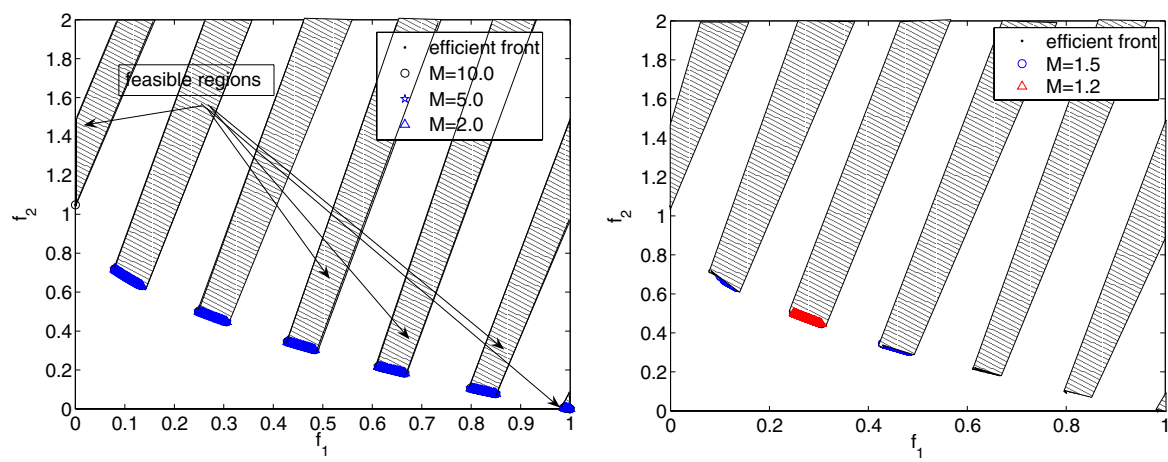

Fig. 6. Proper Pareto optimal solutions Fig. 7. Proper Pareto optimal solutions obtained using $\mathrm{M}=10,5$ and 2 on CTP7 obtained using $\mathrm{M}=1.5$, and 1.2 on CTP7

\section{Acknowledgements}

The author acknowledges the partial financial support by the Gottlieb-Daimlerand Karl Benz-Foundation under Project No. 02-13/05.

\section{References}

1. J. Branke, T. Kaußler, and H. Schmeck. Guidance in evolutionary multi-objective optimization. Advances in Engineering Software, 32:499-507, 2001.

2. K. Deb. Multi-objective optimization using evolutionary algorithms. Chichester, UK: Wiley, 2001.

3. K. Deb, S. Agrawal, A. Pratap, and T. Meyarivan. A fast and elitist multi-objective genetic algorithm: NSGA-II. IEEE Transactions on Evolutionary Computation, 6(2):182-197, 2002.

4. K. Deb, A. Pratap, and T. Meyarivan. Constrained test problems for multi-objective evolutionary optimization. In Proceedings of the First International Conference on Evolutionary Multi-Criterion Optimization (EMO-01), pages 284-298, 2001.

5. Arthur M. Geoffrion. Proper efficiency and the theory of vector maximization. J. Math. Anal. Appl., 22:618-630, 1968.

6. Jen-Chwan Liu. $\epsilon$-properly efficient solutions to nondifferentiable multiobjective programming problems. Appl. Math. Lett., 12(6):109-113, 1999.

7. K. Miettinen. Nonlinear Multiobjective Optimization. Kluwer, Boston, 1999. 